\title{
Chromogranin A - unspecific neuroendocrine marker. Clinical utility and potential diagnostic pitfalls
}

Paweł Gut, Agata Czarnywojtek, Jakub Fischbach, Maciej Bączyk, Katarzyna Ziemnicka, Elżbieta Wrotkowska, Maria Gryczyńska, Marek Ruchała

Department of Endocrinology, Metabolism and Internal Medicine, Poznan University of Medical Sciences, Poznan, Poland

Submitted: 10 January 2014

Accepted: 20 May 2014

Arch Med Sci 2016; 12, 1: 1-9

DOI: 10.5114/aoms.2016.57577

Copyright $\odot 2016$ Termedia \& Banach

\section{Abstract}

Chromogranin A, despite a number of limitations, is still the most valuable marker of neuroendocrine tumors (NETs). Granins belong to the family of acidic proteins that constitute a major component of secretory granules of various endocrine and neuroendocrine cells, which are components of both the classical endocrine glands and the diffuse neuroendocrine system. These cells are a potential source of transformation into neuroendocrine tumors. The awareness of potential causes influencing the false results of its concentrations simplifies diagnosis and treatment. One of the disadvantages of this marker is its non-specificity and the existence of a number of pathological processes leading to an increase in its concentration, which often results in confusion and diagnostic difficulties. The molecular structure is characterized by a number of sites susceptible to the proteolytic activity of enzymes, resulting in the formation of a number of biologically active peptides. Presumably they act as precursors of active proteins. Chromogranin expression correlates with the amount of secretory vesicles in neuroendocrine cells. The peptide chain during biochemical changes becomes a precursor of biologically active proteins with a wide range of activities. There are a number of commercially available kits for the determination of chromogranin A, which differ in methodology. We present the evaluation of chromogranin A as a marker of neuroendocrine tumors in clinical practice and the possible factors that may affect the outcome of its concentration.

Key words: chromogranin A, neuroendocrine tumors.

\section{Introduction}

The growing interest in neuroendocrine tumors, which are interdisciplinary diseases, is associated with an increase in their detection in recent years. Due to their usually indolent character we are dealing with a metastatic proliferative process at the time of diagnosis. Therefore, as in other neoplasms, attempts are made to use biochemical markers that could serve as a valuable diagnostic tool and indicator of response to treatment or recurrence of the disease. The marker for neuroendocrine tumors is chromogranin A, which often in the absence of other biochemical markers of the disease is a useful factor in both the diagnosis and monitoring of disease. One of the disadvantages of this marker is its non-specificity and the existence of a number of pathological processes leading to an increase in its concentration, which often results in confu-

\author{
Corresponding author: \\ Paweł Gut PhD \\ Department of Endocrinology, \\ Metabolism \\ and Internal Diseases \\ University \\ of Medical Sciences \\ 49 Przybyszewskiego St \\ 60-355 Poznan, Poland \\ Phone: +48 618691330 \\ +48607392922 \\ Fax: +48618691682 \\ E-mail: gutpj@poczta.onet.pl
}


sion and diagnostic difficulties. In this review, we would like to present the current state of knowledge about the usefulness of this marker in neuroendocrine tumors and provide a number of conditions that can give false positive results.

\section{General characteristics}

Granins belong to the family of acidic proteins that constitute a major component of secretory granules of various endocrine and neuroendocrine cells, which are components of both the classical endocrine glands and the diffuse neuroendocrine system. These cells are a potential source of transformation into neuroendocrine tumors. The family consists of 8 granin proteins: chromogranin A, B, C (secretogranin II), secretogranin III, IV, V, VI, and VEGF. Granins potentially play an essential role in the creation, maturation and exocytosis of secretory vesicles containing biologically active neuropeptides, neurotransmitters and hormones. Their molecular structure is characterized by a number of sites susceptible to the proteolytic activity of enzymes, resulting in the formation of a number of biologically active peptides. Presumably they act as precursors of active proteins.

Chromogranin A ( $\mathrm{CgA}$ ) was the first identified representative of granins, and derives its name from the spot of primary detection (adrenal medulla chromaffin vesicles containing catecholamines) $[1,2]$. It is encoded by the gene CHGA/CgA located on chromosome 14. Chromogranin expression correlates with the amount of secretory vesicles in neuroendocrine cells. The peptide chain during biochemical changes becomes a precursor of biologically active proteins with a wide range of activities. From the 439-amino acid chain of chromogranin $A$ there arise a number of peptides:

- vasostatin I - exhibiting antiadrenergic activity [3], - parastatin - inhibiting secretion of parathyroid hormone in response to low levels of calcium [4], - pancreastatin - showing a strong inhibitory effect on insulin secretion induced by increasing concentration of glucose in the blood [5],

- catestatin - inhibiting secretion of catecholamines from the chromaffin cells [6].

To date, knowledge about the other products of chromogranin A proteolysis (vasostatin II, chromostatin, GE-25, EC-14) is scant and their biological effect is not certain [7]. Interestingly, proteolysis of chromogranin appears to be organ specific. For example, pancreastatin found in the $\alpha$ cells of the pancreas is absent in adrenal medulla chromaffin cells [8].

\section{Methods for determination of chromogranin}

There are a number of commercially available kits for the determination of chromogranin A, which differ in methodology. Three diagnostic techniques are available: enzyme-linked immunosorbent assay (ELISA), immunoradiometric assay (IRMA) and radioimmunoassay (RIA). There is no standardization for any of the techniques: different studies use different antibodies, which in turn react with different antigenic epitopes on the surface of the protein chain. Determination of CgA by different techniques leads to significant differences in results, with varying effects on sensitivity and specificity. Currently there is no universal, worldwide accepted diagnostic technique; thus caution is recommended when trying to compare the results from different research centers. Chromogranin can be measured from plasma or serum. It has been reported that plasma chromogranin tends to be markedly higher than that determined in the serum [9]. There are many studies comparing the sensitivity and specificity of available diagnostic methods. Stridsberg et al. compared all 3 methods. For IRMA sensitivity was $67 \%$ and specificity $96 \%$, for ELISA sensitivity was $85 \%$ and specificity $85 \%$, and for RIA sensitivity was $93 \%$ and specificity $85 \%$, suggesting that the best compromise between sensitivity and specificity is the use of RIA [10].

\section{Chromogranin A - potentially false positive results}

Because of wide spectrum chromogranin A secretion, together with other regulatory proteins, there are several causes of elevated chromogranin unrelated to neuroendocrine tumor. In clinical practice, the most common causes of false (non-NET) CgA results is the use of proton pump inhibitors, cases of atrophic gastritis and impaired kidney function. The majority of false positive results are about 2-4 times higher than the upper reference range, but a significant number of these results may reach up to 5-20 times higher than the upper reference range [11].

\section{Gastric disorders}

Elevated CgA levels are observed in cases of atrophic gastritis or during treatment with proton pump inhibitors (PPIs) and other acid suppressive medications. Lack of gastric acids engenders hypergastrinemia due to no negative feedback for gastrin, which in turn stimulates the growth of enterochromaffin cells to secrete CgA. Therefore, we obtain elevated circulating CgA. The PPI therapy may increase CgA concentration just 5 days after the first intake and leads to CgA 5-10 times higher than the upper reference range, results often seen in patients with early stage NET [12]. To prevent any impact on the value of CgA by PPIs, a drug should be discontinued at least 7 days before the test. It should also be remembered that the longer 
PPI therapy lasts, the longer is the time needed for the normalization of CgA concentration. Histamine type- 2 receptor antagonists (H2RA) may also have an effect on the increase of the marker. It is suggested to discontinue these medications for at least $24 \mathrm{~h}$ before the scheduled CgA examination.

\section{Impaired kidney function}

Elevated CgA also occurs in the case of impaired kidney function due to reduced renal clearance. The higher the degree of renal failure, the higher the CgA concentration, and the result may reach the level found in neuroendocrine tumors [13]. Before interpreting the result of $\mathrm{CgA}$ as a marker of NET, renal function should be evaluated carefully to rule out the potential impact on the concentration of CgA. Evaluation of CgA as a marker of NET in the case of end stage renal disease is impossible.

\section{Heart diseases}

In a study of 160 patients with chronic heart failure, CgA concentration was significantly higher than in healthy volunteers. CgA levels correlated with the intensity of heart failure defined on the basis of the NYHA scale. Patients in the fourth grade showed the highest concentration of circulating CgA. It was further found that the concentration of chromogranin $A$ is an independent marker of mortality [14]. Interestingly, in patients with chronic heart failure, CgA levels did not correlate with the concentration of the hormones that are involved in the pathophysiological basis of the disease, including catecholamines and components of the renin-angiotensin-aldosterone system [15]. CgA concentration in this case correlated with the concentration of brain natriuretic peptide (BNP) [16, 17]. Elevated chromogranin A is also observed in cases of acute coronary syndromes, where a high concentration of the peptide significantly worsens the prognosis [18]. Circulating CgA seems to reflect inflammation and cardiac overload rather than activation of the autonomous nervous system. In this case, CgA showing a negative inotropic and lusitropic effect plays a protective role in preventing excessive activation of the heart muscle in response to stress factors [16].

\section{Hypertension}

There is a high concentration of CgA in the case of untreated hypertension, and CgA levels may correlate with the severity of hypertension in conjunction with increased adrenergic activity.

\section{Rheumatoid diseases}

In rheumatoid arthritis, the concentration of CgA correlates with the concentration of the receptors for tumor necrosis factor- $\alpha$ (TNF- $\alpha$ ), and treatment with anti-TNF- $\alpha$ impairs this correlation, suggesting a connection between these two molecules. Interestingly, patients with generalized disease manifestations showed unusually high concentrations of CgA, which were sometimes even higher than those observed in patients with neuroendocrine tumors, compared with patients with disease limited only to joints. The CgA concentration seems to reflect generalized inflammation and, by its derivative vasostatin 1 , exhibits a protective effect on the endothelium by inhibiting inflammation mediated by TNF- $\alpha$ [19]. Elevated CgA is also observed in other rheumatoid diseases such as systemic lupus erythematosus.

\section{Inflammatory bowel disease}

Many patients with neuroendocrine tumors derived from the midgut are initially erroneously diagnosed with irritable bowel syndrome, sometimes even a few years before the correct diagnosis. There are reports of an increased concentration of CgA in irritable bowel syndrome and inflammatory bowel disease. Therefore evaluation of CgA is not valuable as a screening test in the evaluation of uncertain cause diarrhea [20]. Approximately $50 \%$ of patients with inflammatory bowel diseases tend to have elevated CgA, especially in the case of the active phase of the disease [21].

\section{Other causes}

Worthy of note is the fact that in both healthy individuals and patients with neuroendocrine tumors CgA increases under the influence of food intake and exercise. Maximum CgA concentrations are observed 30-90 min after a meal and reach 2-3 times the upper reference range [22]. Therefore it is recommended to measure CgA after rest and fasting. Differences in concentration of CgA measured day by day can be up to $20 \%$, and this phenomenon is observed in both healthy volunteers and patients with NET [23]. For other potential causes of false-positive results of chromogranin A see Tables I and II.

\section{Chromogranin A and neuroendocrine tumors}

The CgA is secreted by a number of neuroendocrine tumors, which broadly include: pheochromocytoma, medullary thyroid carcinoma, parathyroid adenomas, pulmonary neuroendocrine tumors including small cell lung cancer, and finally gastroenteropancreatic neuroendocrine tumors (GEP-NETs). Nobels et al. evaluated the usefulness of determining the concentration of chromogranin $A$ as a marker of tumors in different disease entities [24].

The concentration of chromogranin A was elevated in $100 \%$ of cases of gastrinomas, in $89 \%$ of 
Table I. Factors affecting the concentration of chromogranin A

\begin{tabular}{|ll|}
\hline Factor & \multicolumn{1}{c|}{ False positive results } \\
\hline Diseases of the cardiovascular system & Hypertension, heart failure, acute coronary syndrome \\
\hline Renal diseases & Impaired kidney function/renal insufficiency \\
\hline Diseases of the alimentary tract & $\begin{array}{l}\text { Chronic atrophic gastritis, inflammatory bowel diseases, irritable bowel } \\
\text { syndrome, pancreatitis, chronic hepatitis, liver cirrhosis }\end{array}$ \\
\hline Non-neuroendocrine neoplasms & $\begin{array}{l}\text { Prostate cancer, ovarian cancer, breast cancer, colorectal cancer, } \\
\text { pancreatic cancer, hepatocellular carcinoma, hematological malignancies }\end{array}$ \\
\hline Inflammatory diseases & Systemic rheumatoid arthritis, systemic lupus erythematosus, COPD \\
\hline Endocrine disorders & $\begin{array}{l}\text { Pheochromocytoma, hyperparathyroidism, hyperthyroidism, } \\
\text { medullary thyroid cancer, pituitary tumors (except prolactinomas), } \\
\text { hypercortisolemia }\end{array}$ \\
\hline Medications & Proton pump inhibitors, histamine type-2 receptor antagonists \\
\hline Other & Food intake or strenuous exercise before the test \\
\hline
\end{tabular}

Table II. Metabolism of chromogranin A. Neuroendocrine and non-neuroendocrine diseases with elevated chromogranin A concentration in serum

\begin{tabular}{|ll|}
\hline \multicolumn{1}{|c|}{ Neuroendocrine diseases } & \multicolumn{1}{c|}{ Non-neuroendocrine diseases } \\
\hline 1. Pheochromocytoma & 1. Renal failure \\
2. Neuroblastoma & 2. Chronic atrophic gastritis type A \\
3. Medullary thyroid carcinoma & 3. Crohn's disease, ulcerative colitis \\
4. Pituitary adenomas (acromegaly) & 4. Rheumatoid arthritis \\
5. Primary hyperparathyroidism & 5. Parkinson's disease \\
6. Hormonal activity of fetal placenta & 6. Steroid treatment \\
7. Hypercortisolism & \\
\hline
\end{tabular}

pheochromocytomas, $80 \%$ of neuroendocrine tumors of the small intestine, $69 \%$ of non-functioning pancreatic neuroendocrine tumors and in the case of medullary thyroid cancer concentrations above the reference range were recorded in $50 \%$ of cases. What is important, in $7 \%$ of cases in the control group elevated CgA was also observed.

\section{Chromogranin A and non-neuroendocrine neoplasms}

Elevated chromogranin in cases of prostate cancer shows neuroendocrine differentiation of tumor tissue, which is associated with progression, poor prognosis and resistance to hormonal therapy [25]. Elevated concentrations were also observed in the case of other types of cancer: breast cancer, cancer of the digestive gland, cancer of the female genital tract, hematological malignancies and cancers of the head and neck [26]. In studies assessing CgA levels in neoplasms other than neuroendocrine tumors and small cell lung cancer there was no difference between serum CgA and tumor size $[27,28]$. These data are surprising because most tumor markers correlate with tumor size or progression of the disease. Markers are significantly higher in the case of disseminated neoplastic processes. This situation indicates that elevated CgA cannot be used to as- sess the severity or progression of the disease in tumors other than those of neuroendocrine origin.

\section{Chromogranin A and gastroenteropancreatic neuroendocrine tumors}

\section{Chromogranin A concentration and staging}

In general, the highest values of CgA and accuracy in the determination of CgA are observed the most frequently in tumors showing intense secretory activity, mainly neuroendocrine tumors of the small intestine, particularly causing carcinoid syndrome (midgut neuroendocrine tumors) [29]. Chromogranin A is significantly higher in the case of disseminated rather than limited neoplastic disease. An exception may be a gastrinoma, as here CgA is high even in the absence of metastases in the liver [30]. In the case of midgut tumors the comparison of CgA concentration and severity of disease was performed. Patients with multiple liver metastatic disease demonstrated a significantly higher concentration of CgA than patients with only a few lesions in the liver or lymph node metastases [31]. Satisfactory accuracy of CgA is also observed in hormonally inactive cases of neuroendocrine tumors [32]. The CgA is described as a prognostic marker for GEP-NET: high levels correlate with shorter survival and liver cancer bur- 
den. Chromogranin A concentrations and sensitivity depend mainly on the spread of cancer. Arnold et al. reported that the accumulation of metastatic changes in the liver significantly increases the concentration of CgA. In addition, the appearance of metastases to lymph nodes in previously proven metastases in the liver did not cause a further increase in the value of CgA [33]. Moreover, Janson et al. observed a significantly higher CgA concentration in the case of multiple metastatic lesions in the liver $(>5)$ in comparison to the case of only a few metastases $(<5)$ or only lymph metastases [29]. Walter et al. reported that CgA levels are significantly elevated more frequently in the case of NET metastases compared to reduced cancer (74\% vs. $51 \%$ ) [34].

\section{Chromogranin A and tumor primary location and grading}

Taking into account the relationship between the tumor primary location and sensitivity of CgA, most data show higher sensitivity in the case of midgut neuroendocrine tumors compared with pancreatic neuroendocrine tumors $[10,30,35$, 36]. The highest values of CgA are observed in small intestine neuroendocrine tumors (up to 200 times above normal) and GEP-NETs occurring in MEN-1 syndrome (up to 150 times above normal). As regards the pancreatic neuroendocrine tumors, both functioning and non-functioning tumors reveal intermediate levels of CgA (up to 60-80 times the upper reference). In the case of Zollinger-Ellison syndrome connected with MEN1 syndrome, and in the case of type II and III gastric neuroendocrine tumors, values are up to 80-100 times higher than the upper reference range [37]. The sensitivity and specificity of CgA for different types of neuroendocrine tumors are in the range $60-100 \%$ and $70-100 \%$, respectively, with the highest values observed in the case of serotonin-secreting neuroendocrine tumors (carcinoid tumors) $[38,39]$. In the case of serotonin-secreting neuroendocrine tumors originating from the midgut, CgA concentration is an independent prognostic factor, because its concentration is correlated not only with the size of the tumor but also with the intensity of biological activity [40].

\section{Chromogranin A as a prognostic factor}

Chromogranin A concentration can be used as an indirect predictor of cancer. There is a correlation between the staging of the disease and concentration of CgA. As mentioned above, higher CgA is observed for metastatic disease compared to localized disease [41]. There are reports of a correlation between $\mathrm{CgA}$ and survival in neuroendocrine tumors. A concentration 3 times above the upper reference range at the time of diagnosis is a significant predictor of shorter survival (hazard ratio 2.6) in patients with pancreatic neuroendocrine tumors [42]. In the case of neuroendocrine tumors of the midgut, CgA level $>5000 \mu \mathrm{g} / \mathrm{l}$ was an independent predictor of shorter survival of patients, and comparing the results of $>5000 \mu \mathrm{g} / \mathrm{l}$ and < $5000 \mu \mathrm{g} / \mathrm{l}$, the median survival was 33 months and 57 months, respectively [31]. Elevated CgA was associated with significantly shorter survival in the study of midgut tumors with liver metastases treated with a long-acting somatostatin analog, while no relationship between survival and concentrations of urinal 5-hydroxyindoleacetic acid (5-HIAA), the breakdown product of serotonin, was observed [43]. Worthy of note is the fact that the correlation between serum CgA and survival cannot be used generally in relation to GEP-NETs. Gastrinoma are associated with high levels of chromogranin A in serum, even in the absence of metastases in the liver, which is associated with the above-mentioned enterochromaffin cell stimulation by gastrin, in this case with non-negative feedback.

\section{Chromogranin A and relapse of the disease}

In a retrospective study of 56 patients with NET, the concentration of CgA was the first indication of the recurrence of the proliferative process after radically carried out surgery. It suggests that the periodic evaluation of CgA may be a more preferred method than repeated radiological procedures in a situation where we have delayed with complete tumor resection previously [44]. The concentration of chromogranin A correlates with the size and extent of cancer; thus its periodic measurement may be helpful in detecting potential recurrence of the disease. A study evaluating the usefulness of CgA in monitoring of radically treated neuroendocrine tumors showed that the increase in CgA is the first marker of recurrence of the disease, which occurs before changes in the concentration of urinal 5-hydroxyindoleacetic acid or radiologic recurrence [44].

The same study showed that in $86 \%$ of patients with elevated chromogranin $A$ the recurrence of the disease was present subsequently (33 of 56 patients, at a median of 32 months). Another study showed that among patients with GEP-NETs of different locations, who experienced progression of the disease, elevated CgA concentrations were observed in $83 \%$ of cases, while in patients with liver metastasis progression this result was observed in $100 \%$ of cases [45].

\section{Chromogranin $\mathrm{A}$ and response to treatment}

In cases of radically operated neuroendocrine tumors originating from the midgut, CgA levels were recorded as both a diagnostic and early re- 
lapse marker. Reduction of CgA concentration is used frequently as an indicator of response to treatment in clinical trials, in which the biochemical response is usually defined as a $\geq 50 \%$ reduction in the concentration of CgA [33, 44, 46]. It is assumed that the reduction of CgA by more than $50 \%$ may be a proof of the correct choice of treatment. Assuming that the concentration of CgA correlates with the size and number of malignancies, the concentration of CgA is theoretically expected to decrease if treatment is effective. The study by Nehara et al. provided data indicating the reduction of circulating CgA concentration after resection or cytoreduction of the neuroendocrine tumor [41]. In the case of the use of different non-invasive methods also a correlation between the response to treatment of GEP-NET and the concentration of CgA was noted. It occurred as a reduction or stabilization of its concentration [45, 47-49]. Reduction of CgA concentration was observed after successful treatment with radionuclide therapy [50] and after liver transplantation due to massive organ metastases [51]. Generally CgA determination may be a useful tool in monitoring the treatment of GEP-NETs, with the stipulation that an elevated CgA level before therapy was observed [23].

\section{Chromogranin A in the case of somatostatin analog therapy}

In patients treated with somatostatin analogs (SSA), the relationship between circulating CgA and tumor mass is not obvious. This is due to the ability of SSA to block the production and secretion of chromogranin A [52]. Chromogranin A concentration may correlate with tumor size, but care must be taken during interpretation of the results. Serial CgA measurements during follow-up should be performed at approximately the same intervals after the administration of a long-acting SSA.

\section{Chromogranin A and other NET biomarkers}

Chromogranin A can be regarded as an early model of neuroendocrine tumors originating from the foregut and midgut and seems to be a better marker of the disease than the assessment of urinal 5-HIAA and/or circulating serotonin concentrations. There is a stronger correlation between the concentration of CgA compared to 5HIAA with regard to the general condition and well-being of the patient, similar to the correlation between survival and the level of CgA, which makes CgA a recommended first-line marker for GEP-NETs [43]. Another advantage in favor of CgA is definitely an easier way to collect samples for tests, compared to 24-hour 5HIAA urine collection. It should be remembered that in order to avoid false positive results of 5HIAA urine concentration, a special diet excluding numerous sources of tryptophan, such as bananas, cheese, chocolate, tomato, etc., should be introduced. In a study in which 127 patients with neuroendocrine tumors were enrolled, CgA demonstrated superiority over 5HIAA urine concentration, circulating neuron-specific enolase (NSE) and carcinoembryonal antigen (CEA) as a tumor marker [45]. Although the specificity of both 5-HIAA and NSE was high (up to 100\%), low sensitivity (35\% and 33\%, respectively) was observed. Comparing the data for CgA in this case, the specificity was $86 \%$ and sensitivity $68 \%$. It is worth noting that CgA may be a marker for high sensitivity in the case of metastatic NET, but the concentration of 5-HIAA has the highest sensitivity in patients with the symptomatic hormonally active form of GEP-NET [37].

\section{Chromogranin A potentially false-negative results}

Chromogranin A concentration may be normal in the case of neuroendocrine tumors with a mild proliferative potential. These include the majority of neuroendocrine tumors of the appendix, which apart from a few cases are mostly benign tumors, which when totally excised do not require a follow-up after treatment. About $75 \%$ of insulinoma tumors are usually mild and CgA is usually not increased, but in this case the measurement of CgA may be a helpful indicator of tumor malignancy. Besides several typical lung carcinoids, duodenum and rectum low proliferative index tumors may not show elevation of circulating CgA [11]. Rapidly proliferating, poorly differentiated neuroendocrine tumors, which in many cases lose their characteristic structure and show a much smaller number of secretory vesicles, may also not release the marker, giving false negative results.

Although CgA is not a specific marker for particular neuroendocrine tumors, one of its derivatives, neuroendocrine secretory protein 55 (NESP 55), seems to be a specific marker of pheochromocytoma and pancreatic neuroendocrine tumors, neuroendocrine tumors which are not present in the gut [53]. However, so far this marker can be used only with immunohistochemical techniques, and it is impossible to use it as a circulating marker.

\section{Other applications of chromogranin A}

Chromogranin A assessment may be helpful for differentiating sources of high cortisol levels in the case of Cushing's disease between pituitary, adrenal and ectopic ACTH secretion by a neuroendocrine tumor. In the latter case, significantly higher levels of CgA compared to other cases are observed [54]. 
There is a statistically significant difference in the concentration of CgA comparing benign and malignant pheochromocytomas $[55,56]$. The CgA concentrations in the case of malignant lesions were approximately 15 times higher compared to benign lesions. Also there was a positive correlation between CgA and tumor size in the adrenal gland $[57,58]$. Patients with adrenal tumors derived from the adrenal cortex usually do not have elevated circulating chromogranin A levels (especially in the case of incidentalomas) [59]. Chromogranin A concentration correlates well with the production of catecholamines; therefore the determination of CgA is a complementary method to other laboratory tests in the diagnosis of pheochromocytoma such as 24-hour urine catecholamine collection. Determination of CgA concentration is an important tool in the differential diagnosis of pheochromocytoma as a cause of secondary hypertension [58].

\section{Conclusions}

Sensitivity of CgA as a marker of neuroendocrine gastro-entero-pancreatic tumors depends on the location of the original tumor $[60,61]$, metastatic cancer, especially liver metastases $[23,30$, $62,63]$, and laboratory methodology: determining cutoff CgA [10, 34, 41, 62] and the use of a particular method for detection of CgA [10, 36, 62]. The greatest value of CgA as a marker of the disease is achieved in the case of metastatic disease to the liver. It is believed that improvement of the diagnostic sensitivity of CgA can be achieved by combining the measurement of its concentration with another diagnostic technique, especially scintigraphy or PET/CT based on somatostatin and its receptors. It is estimated that determination of CgA concentration and performing receptor scintigraphy with somatostatin analogs increases the sensitivity to $93 \%$, whereas sensitivity of each of these techniques performed separately is estimated at $60-80 \%$ [64]. During the attempt to interpret the result of chromogranin A, caution is recommended, bearing in mind the multitude of factors that may lead to both false positive and negative results.

\section{Conflict of interest}

The authors declare no conflict of interest.

\section{References}

1. Banks P, Helle K. The release of protein from the stimulated adrenal medulla. Biochem J 1965; 97: 40C-1C.

2. Blaschko H, Comline RS, Schneider FH, et al. Secretion of a chromaffin granule protein, chromogranin, from the adrenal gland after splanchic stimulation. Nature 1967; 215: 58-9.
3. Gallo MP, Levi R, Ramella R, et al. Endothelium-derived nitric oxide mediates the antiadrenergic effect of human vasostatin- 1 in the rat ventricular myocardium. Am J Physiol 2007; 292: H2906-12.

4. Fasciotto BH, Trauss CA, Greeley GH, et al. Parastatin (porcine chromogranin A347-419), a novel chromogranin-derived peptide, inhibits paratharoid cell secretion. Endocrinology 1993; 133: 461-6.

5. Helman LJ, Ahn TG, Levine MA, et al. Molecular cloning and primary structure of human chromogranin A (secretory protein I) CDNA. J Biol Chem 1988; 263: 11559-63.

6. Taylor CV, Taupenot L, Mahata SK, et al. Formation of the catecholamine release-inhibitory peptide catestatin from chromogranin A. Determination of proteolytic cleavage sites in hormone storage granules. J Biol Chem 2000; 275: 22905-15.

7. Bilek R, Safarik L, Ciprova P. Chromogranin A, a member of neuroendocrine secretory proteins as a selective marker for laboratory diagnosis of pheochromocytoma. Physiol Res 2008; 57 (Suppl. 1): S171-9.

8. Cetin Y, Aunis D, Bader MF, et al. Chromostatin, a chromogranin A-derived bioactive peptide, is present in human pancreatic insulin (beta) cells. Proc Natl Acad Sci USA 1993; 90: 2360-4.

9. Glinicki P, Kapuścińska R, Jeske W. The differences in chromogranin A (CgA) concentrations measured in serum and in plasma by IRMA and ELISA methods. Endokrynol Pol 2010; 61: 346-50.

10. Stridsberg M, Eriksson B, Oberg K, et al. A comparison between three commercial kits for chromogranin a measurements. J Endocrinol 2003; 177: 337-41.

11. Ardill JE, O'Dorosio TM. Circulating biomarkers in neuroendocrine tumors of the enteropancreatic tract: application to diagnosis, monitoring disease an as prognostic indicators. Endocrinol Metab Clin N Am 2010; 39: 777-90.

12. Pregun I, Herszenyi L, Juhasz M, et al. Effect of proton-pump inhibitor therapy on serum chromogranin A level. Digestion 2011; 84: 22-8.

13. Hsiao RJ, Mezger MS, O'Connor DT. Chromogranin A in uremia: progressive retention of immunoreactive fragments. Kidney Int 1990; 37: 955-64.

14. Ceconi C, Ferrari R, Bachetti T, et al. Chromogranin in heart failure: a novel neurohumoral factor and a predictor for mortality. Eur Heart J 2002; 23: 967-74.

15. Nicholls DP, Onuoha GN, McDowell G, et al. Neuroendocrine changes in chronic cardiac failure. Basic Res Cardiol 1996; 91 (Suppl. 1): 13-20.

16. Pieroni M, Corti A, Tota B, et al. Myocardial production of chromogranin a in human heart: a new regulatory peptide of cardiac function. Eur Heart J 2007; 28: 1117-27.

17. Korse CM, Taal BG, de Groot CA, Bakker RH, Bonfrer JM. Chromogranin-A and N-terminal pro-brain natriuretic peptide: an excellent pair of biomarkers for diagnostics in patients with neuroendocrine tumor. J Clin Oncol 2009; 27: 4293-9.

18. Jansson AM, Rosjo $H$, Omland $T$, et al. Prognostic value of circulating chromogranin A levels in acute coronary syndrome. Eur Heart J 2009; 30: 25-32.

19. DiComte G, Rossi CM, Marinosci A, et al. Circulating chromogranin A reveals extra-articular involvement in patients with rheumatoid arthitis and curbs TNF-alpha-elicited endothelial activation. J Leukoc Biol 2009; 85: 81-7.

20. Sciola V, Massironi S, Conte D, et al. Plasma chromogranin a in patients with inflammatory bowel disease. Inflamm Bowel Dis 2009; 15: 867-71. 
21. Valeur J, Milde AM, Helle KB, et al. Low serum chromogranin $A$ in patients with self-reported food hypersensitivity. Scand J Gastroenterol 2008; 43: 1403-4.

22. Fossmark R, Jianu CS, Martinsen TC, et al. Serum gastrin and chromogranin A levels in patients with fundic gland polyps caused by long-term proton-pump inhibition. Scand J Gastroenterol 2008; 43: 20-4.

23. Lawrence B, Gustafsson BI, Kidd M, et al. The clinical relevance of chromogranin A as a biomarker for gastroenteropancreatic neuroendocrine tumors. Endocrinol Metab Clin N Am 2011; 40: 111-34.

24. Nobels FR, Kwekkeboom BJ, Coopmans W, et al. Chromogranin $A$ as serum marker for neuroendocrine neoplasia: comparison with neuron-specific enolase and the alfa-subunit of glycoprotein hormones. J Clin Endocr Metab 1997; 82: 2622-8.

25. Adolf K, Wagner L, Bergh A, et al. Secretagogin is a new neuroendocrine marker in the human prostate. Prostate 2007; 67: 472-84.

26. Tropea F, Baldari S, Restifo G, et al. Evaluation of chromogranin A expression in patients with non-neuroendocrine tumours. Clin Drug Investig 2006; 26: 715-22.

27. Molina R, Alvarez E, Aniel-Quiroga A, et al. Evaluation of chromogranin A determined by three different procedures in patients with benign diseases, neuroendocrine tumors and other malignancies. Tumor Biol 2011; 32 13-22.

28. Louthan O. Chromogranin A in physiology and oncology. Folia Biologica (Praha) 2011; 57: 173-81.

29. Kos-Kudła B, Bolanowski M, Handkiewicz-Junak D, et al. Diagnostic and therapeutic guidelines for gastrointestinal neuroendocrine tumors (recommended by Polish Network of Neuroendocrine Tumors). Endokrynol Pol 2008; 59: 41-56.

30. Tomasetti P, Migliori M, Simoni P, et al. Diagnostic value of plasma chromogranin $A$ in neuroendocrine tumours. Eur J Gastroenterol Hepatol 2001; 13: 55-8.

31. Janson ET, Holmberg L, Strisberg $M$, et al. Carcinoid tumors: analysis of prognostic factors and survival in 301 patients from a refferal center. Ann Oncol 1997; 8: 685-90.

32. Sobol RE, Memoli V, Deftos LJ. Hormone-negative, chromogranin A-positive endocrine tumors. $N$ Engl J Med 1989; 320: 444-7.

33. Arnold R, Wilke A, Rinke A, et al. Plasma chromogranin $A$ as a marker for survival in patients with metastatic endocrine gastroenteropancreatic tumors. Clin Gastroenterol Hepatol 2008; 6: 820-7.

34. Walter T, Chardon L, Chopin-Laly X, et al. Is the combinantion of chromogranin $A$ and pancreatic polypeptide serum determinations of interest in the diagnosis and follow-up of gastro-entero-pancreatic neuroendocrine tumours? Eur J Cancer 2012; 48: 1766-73.

35. Nobels FR, Kwekkeboom DJ, Bouillon R, et al. Chromogranin A: its clinical value as a marker of neuroendocrine tumours. Eur J Clin Invest 1998; 28: 431-40.

36. Ferrari L, Seregni E, Lucignani G, et al. Accuracy and clinical correlates of two different methods for chromogranin assay in neuroendocrine tumours. Int J Biol Markers 2004; 19: 295-304.

37. Modlin IM, Gustafsson BI, Moss SF, et al. Chromogranin A- biological function and clinical utility in neuro endocrine tumor disease. Ann Surg Oncol 2010; 17: 2427-43.

38. Vinik AI, Silva MP, Woltering G, et al. Biochemical testing for neuroendocrine tumors. Pancreas 2009; 38: 876-89.

39. O'Toole D, Grossman A, Gross D, et al. ENETS Consensus Guidelines for the Standards of Care in Neuroendocrine Tumors: biochemical markers. Neuroendocrinology 2009; 90: 194-202.
40. Gustafsson BI, Kidd M, Modlin IM. Neuroendocrine tumors of the diffuse neuroendocrine system. Curr Opin Oncol 2008; 20: 1-12.

41. Nehar D, Lombard-Bohas C, Olivieri S, et al. Serum chromogranin $A$ for diagnosis and follow-up of endocrine tumors. Clin Endocrinol (Oxf) 2004; 60: 644-52.

42. Ekeblad S, Skogseid B, Dunder K, et al. Prognostic factors and survival in 324 patients with pancreatic endocrine tumours treated at a single institution. Clin Cancer Res 2008; 14: 7789-803.

43. Korse CM, Bonfrer JM, Aaronson NK, et al. Chromogranin $\mathrm{A}$ as an alternative to 5 -hydroxyindolaecetic acid in the evaluation of symptoms during treatment of patients with neuroendocrine tumors. Neuroendocrinology 2009; 89: 296-301.

44. Welin S, Strisberg M, Cunningham J, et al. Elevated plasma chromogranin $A$ is the first indication of recurrence in radically operated midgut carcinoid tumors. Neuroendocrinology 2009; 89: 302-7.

45. Bajetta E, Ferrari L, Martinetti A, et al. Chromogranin, neuron specific enolase, carcinoembryonic antigen and hydroxyindole acetic acid evaluation in patients with neuroendocrine tumors. Cancer 1999; 86: 858-65.

46. Eriksson B, Arnberg H, Oberg K, et al. Chromogranins new sensitive markers for neuroendocrine tumors. Acta Oncol 1989; 28: 325-9.

47. Granberg D, Wilander E, Stridsberg $M$, et al. Clinical symptoms, hormone profiles, treatment and prognosis with gastric carcinoids. Gut 1998; 43: 223-8.

48. Stivanello $M$, Berutti A, Torta $M$, et al. Circulating chromogranin $A$ in the assessment of patients with neuroendocrine tumours. A single institution experience. Ann Oncol 2001; 12 (Suppl. 2): S73-7.

49. Shojamanesh H, Gibril F, Louie A, et al. Prospective study of the antitumor efficacy of long-term octreotide treatment in patients with progressive metastatic gastrinoma. Cancer 2002; 94: 331-43.

50. Kwekkeboom DJ, Teunissen JJ, Bakker WH, et al. Radiolabeled somatostatin analog [177-Lu-DOTA0,Tyr3]octreotate in patients with endocrine gastroenteropancreatic tumors. J Clin Oncol 2005; 23: 2754-62.

51. Olausson M, Friman S, Herlenius G, et al. Orthotropic liver or multivisceral transplantation as treatment of metastatic neuroendocrine tumors. Liver Transpl 2007; 13: 327-33.

52. Rinke A, Muller $\mathrm{HH}$, Schade-Brittinger $\mathrm{C}$, et al. Placebocontrolled, double-blind, prospective, randomized study on effect of octreotide LAR in the control of tumor growth in patients with metastatic neuroendocrine midgut tumors: a report from the PROMID Study Group. J Clin Oncol 2009; 27: 4656-63.

53. Srivastava A, Padilla O, Fischer-Colbrie R, et al. Neuroendocrine secretory protein-55 (NESP-55) expression discriminates pancreatic endocrine tumors and pheochromocytomas from gastrointestinal and pulmonary carcinoids. Am J Surg Pathol 2004; 28: 1371-8.

54. Nobels FR, de Herder WW, Kwekkeboom DJ, et al. Serum chromogranin $A$ in the differential diagnosis of Cushing's syndrome. Eur J Endocrinol 1994; 131: 589-93.

55. Feng C, Li HZ, Yan WG, et al. The expression and significance of chromogranin $A$ and synaptophysin in adrenal gland tumors. Zhonghua Zhong Liu Za Zhi 2005; 27: 486-8

56. Portela-Gomes GM, Stridsberg M, Grimelius L, et al. Expression of chromogranins $A, B$, and $C$ (secretogranin II) in human adrenal medulla and in benign and malig- 
nant pheochromocytomas. An immunohistochemical study with region-specific antibodies. APMIS 2004; 112: 663-73

57. Giovanella L, Squin N, Ghelfo A, et al. Chromogranin A immunoradiometric assay in diagnosis of pheochromocytoma: comparison with plasma metanephrines and 123I-MIBG scan. Q J Nucl Med Mol Imaging 2006; 50: 344-7.

58. D’Herbomez M, Gouze V, Huglo D, et al. Chromogranin A assay and (131)I-MIBG scintigraphy for diagnosis and follow-up of pheochromocytoma. J Nucl Med 2001; 42: 993-7.

59. Bernini GP, Moretti A, Borgioli M, et al. Plasma and tissue chromogranin in patients with adrenocortical adenomas. J Endocrinol Invest 2004; 27: 821-5.

60. Schurman G, Raeth U, Wiedenmann B, et al. Serum chromogranin $A$ in the diagnosis and follow-up of neuroendocrine tumors of the gastroenteropancreatic tract. World J Surg 1992; 16: 697-701.

61. Baudin E, Bihart JM, Bachelot A, et al. Impact of chromogranin A measurement in the work-up of neuroendocrine tumors. Ann Oncol 2001; 12: S79-82.

62. Zatelli MC, Torta M, Leon A, et al. Chromogranin A as a marker of neuroendocrine neoplasia. An Italian Multicenter Study. Endocr Relat Cancer 2007; 14: 473-82.

63. Nikou GC, Marinou K, Thomakos P, et al. Chromogranin A levels in diagnosis treatment and follow up of 42 patients with non functioning pancreatic endocrine tumors. Pancreatology 2008; 8: 510-9.

64. Namwongprom S, Wong FC, Tateishi U, et al. Correlation of chromogranin A levels and somatostatin receptor scintigraphy findings in the evaluation of metastases in carcinoid tomours. Ann Nucl Med 2008; 22: 237-43. 